PROMOCIÓN DE LA RESPONSABILIDAD SOCIAL EN EMPRESAS MANUFACTURERAS EXPORTADORAS DEL ESTADO DE HIDALGO PARA EL DESARROLLO REGIONAL

\title{
PROMOCIÓN DE LA RESPONSABILIDAD SOCIAL EN EMPRESAS MANUFACTURERAS EXPORTADORAS DEL ESTADO DE HIDALGO PARA EL DESARROLLO REGIONAL
}

\section{SOCIAL RESPONSIBILITY PROMOTION IN EXPORTING AND MANUFACTURING COMPANIES FROM HIDALGO FOR THE REGIONAL DEVELOPMENT}

\author{
Eduardo Muñoz Bautista*, Arlén Cerón Islas**, Iván Hernández Ortiz***, \\ Sofía Emilia López García****
}

* Doctor en Dirección y Mercadotecnia y Doctor en Desarrollo Económico y Sectorial Estratégico. Universidad Autónoma del Estado de Hidalgo; Instituto de Ciencias Económico Administrativas; Académico tiempo completo, Área académica de Mercadotecnia.

** Doctora en Planeación Estratégica y Dirección de Tecnología. Universidad Autónoma del Estado de Hidalgo; Instituto de Ciencias Económico Administrativas; Académico tiempo completo, Área académica de Mercadotecnia. Email: arlenceis@gmail.com

*** Doctor en Planeación Estratégica y Dirección de Tecnología. Universidad Autónoma del Estado de Hidalgo; Instituto de Ciencias Económico Administrativas; Académico tiempo completo, Área de Mercadotecnia. Email: ivan_hernandez_ortiz@hotmail.com

**** Maestra en Psicopedagogía. Universidad Autónoma del Estado de Hidalgo; Instituto de Ciencias Económico Administrativas; Académico tiempo parcial, Área de Comercio Exterior y Mercadotecnia. Email: sofialg2002@hotmail.com

Dirección para recibir correspondencia: eduardo_munoz5599@uaeh.edu.mx

Fecha de recibido: 29 de enero de 2018

Fecha de aceptación: 20 de marzo de 2018 MUÑOZ-BAUTISTA E., CERÓN-ISLAS A., HERNÁNDEZ-ORTIZ I., LÓPEZ-GARCÍA S. E. 
PROMOCIÓN DE LA RESPONSABILIDAD SOCIAL EN EMPRESAS MANUFACTURERAS EXPORTADORAS DEL ESTADO DE HIDALGO PARA EL DESARROLLO REGIONAL

\section{RESUMEN}

El presente artículo, es parte del resultado de la investigación "La responsabilidad social empresarial en empresas exportadoras manufactureras del Estado de Hidalgo".

OBJETIVO: Identificar la opinión de empleados hacia las acciones y requerimientos de responsabilidad social empresarial para realizar propuesta de modelo de RS.

MATERIAL Y MÉTODO: El diseño de la investigación es transversal y correlacional, considerando una muestra de 25 empresas activas de 38 en el ramo, mediante el muestreo probabilístico por áreas y polietápico ponderando, respectivamente en 4 municipios, el método de recolección de la información fue mediante una encuesta aplicada a 25 empleados de nivel directivo y 250 de nivel operativo.

RESULTADOS: Los directivos se identificaron en apoyos al conocimiento de la RS apenas si el $56 \%$ lo considera en referencia asesoría, mientras que el $64 \%$ considera apoyos en cuanto a capacitación y elementos tecnológicos.

CONCLUSIONES: De los requerimientos para la aplicación de la Responsabilidad Social, se consideran dos dimensiones: apoyos al conocimiento RS y apoyos requeridos de otros organismos. Este documento puntualiza la promoción para implementar la responsabilidad social en las empresas manufactureras exportadoras que contribuyan al desarrollo regional del Estado de Hidalgo de manera sustentable.

PALABRAS CLAVE: Empresas exportadoras. Responsabilidad social empresarial. Desarrollo regional.

\section{ABSTRACT}

This article is part of the research "Corporate Social Responsibility" in exporting and manufacturing companies from Hidalgo".

OBJECTIVE: To identify employees' opinion towards CSR actions and requirements in order to create a SR Model proposal. 
PROMOCIÓN DE LA RESPONSABILIDAD SOCIAL EN EMPRESAS MANUFACTURERAS EXPORTADORAS DEL ESTADO DE HIDALGO PARA EL DESARROLLO REGIONAL

MATERIAL AND METHOD: This is a cross-sectional and correlational study, with a 25 active companies out of 38 as a sample, selected through areas and a multi-stage sampling, in 4 municipalities respectively. The data collection instrument was a survey applied to 25 management level employees and 250 operative level employees.

RESULTS: Barely the $56 \%$ of the management level employees identified themselves in support to SR knowledge and consider it consulting, while the $64 \%$ consider it as a support in training and in technological elements.

CONCLUSIONS: Two dimensions are considered for the application of SR requirements: support to SR knowledge and needed support from other organizations. This document points out promotion as a means of implementing Social Responsibility in exporting and manufacturing companies that contribute to the regional development in a sustainable way in the state of Hidalgo.

KEYWORDS: Exporting companies. Corporate social responsibility. Regional development.

\section{INTRODUCCIÓN}

Actualmente, se ha ingresado en el período donde las organizaciones públicas o privadas deben establecer una estrategia bien estructurada sobre la base de la Responsabilidad Social Empresarial (RSE) y no sólo realizar acciones aisladas de filantropía (Díaz, 2011), el desarrollo de prácticas de (RSE) implica un aprendizaje y la capacidad de entender el contexto de las partes (Lindgreen, Swaen y Johnston, 2009). Pedroza y Ahuja (2008) establecen que "en la medida que la empresa asume la RSE, camina hacia un desarrollo sustentable de su actividad productiva" ( $p$. 38), los mismos consideran que la RSE "no es algo añadido, sino que es parte de la empresa y requiere de una visión integral, así mismo es parte del (core business)" (p. 39), denominación inglesa que refiere al corazón del negocio la cual es una herramienta necesaria para establecer una ventaja competitiva.

A nivel internacional, México ha tenido un enfoque limitado en materia de responsabilidad corporativa y presentación de sus informes (KPMG, 2011), claro está que las empresas en la región del Estado de Hidalgo no refleja informes de comunicación en materia de ser empresa 
PROMOCIÓN DE LA RESPONSABILIDAD SOCIAL EN EMPRESAS MANUFACTURERAS EXPORTADORAS DEL ESTADO DE HIDALGO PARA EL DESARROLLO REGIONAL

socialmente responsable, al respecto Trejo (2012) sostiene que en la región del Estado de Hidalgo son sólo cuatro empresas las que reportan su responsabilidad social, así mismo Muñoz y López (2013) identificaron que de 40 empresas exportadoras sólo tres reportan sus actividades, en este contexto, dos de ellas son manufactureras y una del área comercial, siendo el total de 37 empresas que no reportan su responsabilidad social 36 del ramo manufacturero y una del comercial (aclarando que los autores no retoman en su estudio las empresas de servicio). Lo anterior, refleja que en el Estado de Hidalgo es muy bajo el número de empresas que reportan su responsabilidad social.

Las empresas exportadoras deberán orientar sus esfuerzos hacia estrategias de competitividad que reflejen la (RSE) y establecer una cultura hacia un desarrollo económico sustentable que beneficie a sus entornos: internos y externos. Para lo cual se planteó como objetivo de investigación: Identificar la opinión de empleados hacia las acciones y requerimientos de responsabilidad social empresarial en empresas manufactureras exportadoras del Estado de Hidalgo, con la finalidad de desarrollar un modelo de RS para el desarrollo regional de manera sustentable, delimitando el estudio en los municipios de Pachuca de Soto, Tulancingo de Bravo, Tepeapulco y Tizayuca.

\section{MATERIAL Y MÉTODO}

La metodología parte de la hipótesis, las empresas manufactureras exportadoras del Estado de Hidalgo de los municipios de Pachuca de Soto, Tulancingo de Bravo, Tepeapulco y Tizayuca, consideran necesario el apoyo externo para implementar su responsabilidad social de acuerdo a la percepción de los empleados de nivel directivo y operativo, valida y facilita la realización de un modelo para implementar la responsabilidad social empresarial que contribuyan al desarrollo regional de manera sustentable.

Por lo que para realizar la comprobación de la misma, se establece el diseño de la investigación tipo transversal debido a que se recopilan datos en un momento único, cuyo propósito es describir variables y analizar su incidencia e interrelación en un momento dado, de igual forma se torna correlacional debido a que se establecen relaciones entre dos o más categorías en un momento determinado (Hernández, Fernández y Baptista, 2010). Es de corte cuantitativo, teniendo como muestra a 25 empresas exportadoras manufactureras de 36 en el Estado de Hidalgo, obteniendo información de 250 empleados de nivel operativo y 25 directivos en los municipios de Pachuca de Soto, Tulancingo de Bravo, Tepeapulco y Tizayuca, ya que es donde se encuentran más MUÑOZ-BAUTISTA E., CERÓN-ISLAS A., HERNÁNDEZ-ORTIZ I., LÓPEZ-GARCÍA S. E. 
PROMOCIÓN DE LA RESPONSABILIDAD SOCIAL EN EMPRESAS MANUFACTURERAS EXPORTADORAS DEL ESTADO DE HIDALGO PARA EL DESARROLLO REGIONAL

concentradas este tipo de empresas en el Estado de Hidalgo (Muñoz y López, 2013), la elección de los encuestados fue de manera aleatoria por conveniencia; dicha encuesta fue aplicada en el segundo semestre del 2017.

Para la obtención de la información fue mediante una encuesta, utilizando un cuestionario con preguntas de escala de Likert con cinco categorías de respuesta de uso muy definido, con totalmente en desacuerdo $1,2,3,4,5$, a totalmente de acuerdo, donde se requiere que los encuestados indiquen el que más se acerca a su realidad (Malhotra, 2008), basado en requerimientos para la aplicación de la responsabilidad social (RS), haciendo análisis descriptivo e inferencial univariado chi cuadrada utilizando el programa estadístico Statistical Package for the Social Sciences (SPSS) por sus siglas en ingles versión 21. En la operacionalización del constructo "los requerimientos para aplicación de la RS" se identifican dos dimensiones 1) apoyos al conocimiento de responsabilidad social, y 2) apoyos requeridos de otros organismos cada una con sus respectivas variables apoyados con los autores que la sostienen su marco teórico.

Se realiza un Análisis inferencial univariado, chi cuadrada (de Pearson) por tipo de empleado; considerando que los métodos estadísticos univariados ofrecen mayor exactitud en el análisis, utilizando el programa estadístico SPSS V-21 se procedió a realizar:

- La tabulación cruzada, como una técnica estadística que describe dos variables simultáneamente y que a través de tablas de contingencia se muestra la distribución conjunta de dos variables, que tienen un número limitado de categorías o valores distintos y son llamadas también tablas de contingencia.

- Chi cuadrada (de Pearson) que en esta investigación permite comprobar si en una tabla de contingencia, una de las variables influye sobre la otra.

El nivel de significancia utilizado en esta investigación es $\alpha=0,05$; como se sabe, si un contraste de hipótesis proporciona un valor " $p$ " inferior a " $\alpha$ " la hipótesis nula es rechazada, considerando el resultado de manera significativa, cuanto menor sea el valor de $\mathrm{P}$ será más significativo el resultado (Devore, 2001). La hipótesis es la proposición no probada que explica tentativamente ciertos hechos o fenómenos, diferenciando de dos maneras la hipótesis nula: siendo el Estado en que se encuentran las cosas y en el que no se espera ninguna diferencia ni efecto, cuyo símbolo es "Ho" y la hipótesis alternativa siendo un enunciado en el que se espera alguna diferencia o efecto y su símbolo es "H1" (Hernández et al, 2010). 
PROMOCIÓN DE LA RESPONSABILIDAD SOCIAL EN EMPRESAS MANUFACTURERAS EXPORTADORAS DEL ESTADO DE HIDALGO PARA EL DESARROLLO REGIONAL

El coeficiente de correlación no paramétrico Gamma de Goodman-Kruskal, mide la relación bivariada entre una variable ordinal y una nominal, considerando los siguientes intervalos para su interpretación:

0.01 a 0.09 - asociación insignificante.

0.10 a 0.29 - asociación baja.

0.30 a 0.49 - asociación moderada.

0.50 a 0.69 - asociación substancial; 0.70 o mayor asociación muy fuerte.

El análisis de todos los ítems del instrumento aplicado, cada una de ellos fungen como variable dependiente y como variable independiente los tipos de empleado (directivos y operativos).

\section{Marco teórico}

El desarrollo esquemático de las intenciones, las acciones de responsabilidad social y los apoyos externos para implementarla, la posición de las empresas manufactureras exportadoras del Estado de Hidalgo fue de relevancia, ya que el hecho de considerar por una parte las intenciones: elementos de comportamiento basado en la parte de la conciencia y en la reglamentación. Son diversas teorías que sostienen dicho estudio. A tal efecto, la teoría de la ética de las intenciones determina que una acción es definida como moralmente buena, atendiendo a dos reglas la próxima (la conciencia) y la remota (la ley), por lo tanto, la intención es la que importa la que hace beneficiosa la manera en que la riqueza es producida (Calvo 2006: 9). Otra teoría es la del desarrollo regional, que sostiene que el desarrollo como concepto ha evolucionado, y se define como un concepto integral, dependiendo de las capacidades de la gente, además de las capacidades productivas e institucionales de la región o país (Flores, 2012). Por su parte, Delgadillo, Torres y Gazca (2001) consideran que el desarrollo regional es un proceso y un fin en las tareas de administración y promoción del crecimiento y el bienestar del país.

Se retoma la teoría de la base de exportación acentúa la importancia del sector exportador en el desarrollo regional, ya que la base de exportación de una región la conforman la actividad exportadora ya sea primeria, secundaria o terciaria (Salguero, 2006). Asimismo, se sustenta con la teoría de la ventaja competitiva a nivel nacional, establece la capacidad de las industrias de un país para ser innovadoras y cambiar a un nivel más elevado de tecnología y productividad (Rachman, 1996). A nivel empresa, es cuando logra más valor para los clientes que sus 
PROMOCIÓN DE LA RESPONSABILIDAD SOCIAL EN EMPRESAS MANUFACTURERAS EXPORTADORAS DEL ESTADO DE HIDALGO PARA EL DESARROLLO REGIONAL

competidores (Keegan y Green, 2009). Para tener éxito en los mercados internacionales las empresas obtienen ventaja mediante actos de innovación, incluyendo nuevas tecnología y nuevas maneras de hacer las cosas (Porter, 2008). Teoría relativa a la estructura industrial regional, de acuerdo al autor Richardson citado por Salguero (2006) establece "las interrelaciones del desarrollo económico de una región con su estructura industrial y los cambios de la misma" (p. 17) a partir del análisis de la estructura industrial de una región como componente de la economía nacional, se puede denotar el cambio hacia un desarrollo regional.

Los diversos organismos preocupados por el desarrollo sustentable de la actividad productiva y comercial, deben de estar vinculados, entre los que destacan:

Organismos públicos descentralizados: En el Estado de Hidalgo se encuentra la Corporación Internacional Hidalgo (COINHI) que manifiesta proporcionar asistencia, apoyo integral y asesoría estratégica a las empresas interesadas en exportar productos hidalguenses que contribuyan al desarrollo económico y sustentable del Estado de Hidalgo (Lugo, 2011).

Agencias de cooperación internacional: Entre ellas resalta la agencia de cooperación técnica alemana (GTZ) por sus siglas en alemán, es una empresa de cooperación internacional para el desarrollo sostenible. Ofrece soluciones con proyección de futuro para el desarrollo político, económico, ecológico y social en un mundo globalizado y fomenta, incluso bajo condiciones difíciles, procesos complejos de cambio y de reformas, siendo su objetivo el de mejorar de forma sostenible las condiciones de vida de las personas (Korin, 2013).

Organismos empresariales: En México se conocen como cámaras y confederaciones que son instituciones de interés público, con autonomía, no lucrativas, con personalidad jurídica propia y que están integradas por industriales, regidas por la Ley de Cámaras y sus Confederaciones, que son organismos para apoyar el desarrollo empresarial, como la (Cámara Nacional de la Industria de Transformación [CANACINTRA], 2013).

Institutos de investigación y/o Universidades: Brindan el recurso humano capacitado en diversas áreas del conocimiento que se requieren en la aplicación y reportes de las acciones de la responsabilidad social. Tales como la Universidad Autónoma del Estado de Hidalgo (UAEH) que establece a través de la Dirección de vinculación con el sector social y productivo "Motiva e induce al desarrollo de habilidades emprendedoras en los estudiantes para proyectos productivos y de negocio, de investigación, de comunicación, del cuidado del medio ambiente y de responsabilidad 
PROMOCIÓN DE LA RESPONSABILIDAD SOCIAL EN EMPRESAS MANUFACTURERAS EXPORTADORAS DEL ESTADO DE HIDALGO PARA EL DESARROLLO REGIONAL

social" (UAEH, 2013, p. 1); Asimismo, la Dirección de servicio social y prácticas profesionales, plantea sus objetivos específicos de cada una de estas actividades, tal es el caso del servicio social universitario el cual pretende vincular la labor de los (las) prestadores (as) de servicio de todas las licenciaturas con la realidad social mexicana para dar respuesta comprometida a la población más necesitada del país a través de un trabajo profesional que fortalezca el vínculo universidad-sociedad, en el caso de las prácticas profesionales pretende vincular al alumno (a) en escenarios reales que le permitan realizar sus prácticas profesionales para fomentar el desarrollo de sus diversas competencias (UAEH, 2014).

Organismos del gobierno federal. Entre ellos se encuentra PROMÉXICO que es el encargado de fortalecer la participación de México en la economía internacional dentro de sus objetivos se identifica el de "promover y apoyar la actividad exportadora y la internacionalización de las empresas mexicanas, a través de medidas que reordenen, refuercen, mejores e innoven los mecanismos de fomento a la oferta exportable competitiva" (PROMÉXICO, 2013, p. 1). Pretende incentivar a las empresas que comuniquen su responsabilidad social a través de:

- Apoyos de capacitación y asesoría con costos de cuota de recuperación.

- Apoyos de financiamiento a las exportaciones.

En cuanto a las acciones de responsabilidad social diferentes organismos como: World Confederation of Businesses, Global Reporting Initiative, Organización Internacional de Normalización, NMX-SAST-26000-IMNC-2011, entre otros que evalúan el desempeño mediante metodologías diversas pero afines, siendo de valiosa aportación para la apreciación en las empresas manufactureras exportadoras del Estado de Hidalgo concretándose en: actividades primarias y de soporte, prácticas justas de operación, iniciativas ambientales, compromiso social con la comunidad, factor humano, prácticas laborales y respeto a los consumidores (WORLDCOB, 2011; GRI, 2006; ISO, 2009; DOF, 2012).

\section{RESULTADOS}

En cuanto al análisis "requerimientos para la aplicación de la Responsabilidad Social" desde la perspectiva de sus empleados de nivel directivo y de nivel operativo. Los directivos se identificaron en apoyos al conocimiento de la RS apenas si el 56\% lo considera en referencia asesoría, mientras que el $64 \%$ considera apoyos en cuanto a capacitación y elementos tecnológicos. Los de nivel operativo el $69 \%$ consideran que sus empresas requieren asesoría y 
PROMOCIÓN DE LA RESPONSABILIDAD SOCIAL EN EMPRESAS MANUFACTURERAS EXPORTADORAS DEL ESTADO DE HIDALGO PARA EL DESARROLLO REGIONAL

capacitación para aplicar acciones de responsabilidad social, así como el 74\% consideraron que sus empresas requieren de tecnología para el fin mencionado. Se observa que las puntuaciones medias están sesgadas hacia la derecha presentando dispersiones altas. Determinando que más de la mitad requieren apoyo en capacitación y elementos tecnológicos para difundir su responsabilidad social (ver tabla 1 y 2 ).

En cuanto a los apoyos requeridos por otros organismos para aplicar la responsabilidad social en las empresas manufactureras exportadoras del Estado de Hidalgo, desde la opinión del empleado de nivel directivo y operativo, en referencia a los directivos, se ubica que más del $50 \%$ lo aceptan y poco menos de la mitad no lo consideran necesario, puntualizando que el $72 \%$ estableció necesitar apoyo de PROMÉXICO y el 64\% de COINHI, el 60\% a los organismos empresariales, dejando en el $52 \%$ a las agencias de cooperación internacional y a los institutos de investigación y/o universidades. Los de nivel operativo en promedio de un $66 \%$ que sus empresas requieren de apoyo como PROMÉXICO, institutos de investigación y/o universidades, cámaras y/o confederaciones, organismos públicos descentralizados y sólo un $63 \%$ de agencias de cooperación internacional, como se puede observar en la tabla 1.

Los datos estadísticos en general las puntuaciones medias están sesgadas hacia la derecha y las dispersiones se ubican altas. Lo que indica, que las empresas manufactureras exportadoras del Estado de Hidalgo desde la perspectiva de sus directivos y operativo, aceptan más el apoyo de PROMÉXICO como dependencia gubernamental federal, así como de organismos descentralizados (ver tabla 1 y 2 ). 
PROMOCIÓN DE LA RESPONSABILIDAD SOCIAL EN EMPRESAS MANUFACTURERAS EXPORTADORAS DEL ESTADO DE HIDALGO PARA EL DESARROLLO REGIONAL

Tabla 1

Descriptivos de requerimientos para la comunicación de RS (ejecutivos)

Apoyos al conocimiento de RS

\begin{tabular}{|c|c|c|c|c|c|c|}
\hline & $\begin{array}{l}\text { Frecuencias } \\
\text { Totalmente en } \\
\text { desacuerdo, } \\
\text { desacuerdo, ni } \\
\text { de acuerdo ni } \\
\text { desacuerdo }\end{array}$ & $\%$ & $\begin{array}{l}\text { De acuerdo, } \\
\text { totalmente } \\
\text { de acuerdo }\end{array}$ & $\%$ & Estadísti & D. E. \\
\hline $\begin{array}{l}72 \text { ¿La empresa requiere asesoría para } \\
\text { comunicar acciones de su } \\
\text { responsabilidad social? } \\
73 \text { ¿La empresa requiere capacitación }\end{array}$ & 11 & 44.0 & 14 & 56.0 & 3.52 & 1.046 \\
\hline $\begin{array}{l}\text { para comunicar acciones de su } \\
\text { responsabilidad social? }\end{array}$ & 9 & 36.0 & 16 & 64.0 & 3.48 & 1.085 \\
\hline $\begin{array}{l}74 \text { ¿La empresa requiere de elementos } \\
\text { tecnológicos para comunicar acciones de } \\
\text { su responsabilidad social? }\end{array}$ & 9 & 36.0 & 16 & 64.0 & 3.80 & 1.000 \\
\hline Apoyos requeridos de otros organ & smos & & & & & \\
\hline $\begin{array}{l}75 \text { ¿La empresa requiere de apoyo de } \\
\text { las dependencias de gobierno federal } \\
\text { como PROMEXICO para comunicar } \\
\text { acciones de su responsabilidad social? }\end{array}$ & 7 & 28.0 & 18 & 72.0 & 3.80 & 1.155 \\
\hline $\begin{array}{l}76 \text { ¿La empresa requiere de apoyo de } \\
\text { los institutos de investigación y/o } \\
\text { universidades para comunicar acciones } \\
\text { de su responsabilidad social? }\end{array}$ & 12 & 48.0 & 13 & 52.0 & 3.44 & 1.227 \\
\hline $\begin{array}{l}77 \text { ¿La empresa requiere de apoyo de } \\
\text { los organismos empresariales como las }\end{array}$ & & & & & & \\
\hline $\begin{array}{l}\text { cámaras y/o confederaciones para } \\
\text { comunicar acciones de su } \\
\text { responsabilidad social? }\end{array}$ & 10 & 40.0 & 15 & 60.0 & 3.72 & 1.100 \\
\hline $\begin{array}{l}78 \text { ¿La empresa requiere de apoyo de } \\
\text { los organismos públicos descentralizados } \\
\text { como la COINHI para comunicar } \\
\text { acciones de su responsabilidad social? }\end{array}$ & 9 & 36.0 & 16 & 64.0 & 3.52 & 1.358 \\
\hline $\begin{array}{l}79 \text { ¿La empresa requiere de apoyo de } \\
\text { agencias de cooperación internacional } \\
\text { para comunicar acciones de su } \\
\text { responsabilidad social? }\end{array}$ & 12 & 48.0 & 13 & 52.0 & 3.32 & 1.180 \\
\hline
\end{tabular}

Fuente: Elaboración propia, 2017.

MUÑOZ-BAUTISTA E., CERÓN-ISLAS A., HERNÁNDEZ-ORTIZ I., LÓPEZ-GARCÍA S. E.

MAYO-AGOSTO 2018. Año 24, Número 69. Págs. 425-442 
PROMOCIÓN DE LA RESPONSABILIDAD SOCIAL EN EMPRESAS MANUFACTURERAS EXPORTADORAS DEL ESTADO DE HIDALGO PARA EL DESARROLLO REGIONAL

\section{Tabla 2}

Descriptivos de requerimientos para la comunicación de RS (operativos)

Apoyos al conocimiento de RS

72 ¿La empresa requiere asesoría para

comunicar acciones de su responsabilidad social?

73 ¿La empresa requiere capacitación para comunicar acciones de su responsabilidad social?

74 ¿La empresa requiere de elementos tecnológicos para comunicar acciones de su responsabilidad social?

Apoyos requeridos de otros organismos

75 ¿La empresa requiere de apoyo de las dependencias de gobierno federal como PROMEXICO para comunicar acciones de su responsabilidad social?

76 ¿La empresa requiere de apoyo de los institutos de investigación y/o universidades para comunicar acciones de su responsabilidad social?

77 ¿La empresa requiere de apoyo de los organismos empresariales como las

cámaras y/o confederaciones para comunicar acciones de su responsabilidad social?

78 ¿La empresa requiere de apoyo de los organismos públicos descentralizados como la COINHI para comunicar acciones de su responsabilidad social? 79 ¿La empresa requiere de apoyo de agencias de cooperación internacional para comunicar acciones de su responsabilidad social?
81

Frecuencias
Totalmente en
desacuerdo, en
desacuerdo, ni de
acuerdo ni desacuerdo

79

78

65

76

87

91

90
Frecuencias

$\mathrm{De}$

$\%$

$$
\text { acuerdo, }
$$
totalmente

$\%$

Media

D. E.

de acuerdo

31.6

171

68.4

3.76

1.074

31.2

172

68.8

3.82

1.061

26.0

185

74.0

.965

169

$67.6 \quad 3.80$

1.034

36.0

160

64.0

3.74

1.013

Fuente: Elaboración propia, 2017.

MUÑOZ-BAUTISTA E., CERÓN-ISLAS A., HERNÁNDEZ-ORTIZ I., LÓPEZ-GARCÍA S. E.

MAYO-AGOSTO 2018. Año 24, Número 69. Págs. 425-442 
PROMOCIÓN DE LA RESPONSABILIDAD SOCIAL EN EMPRESAS MANUFACTURERAS EXPORTADORAS DEL ESTADO DE HIDALGO PARA EL DESARROLLO REGIONAL

Análisis de los requerimientos para la aplicación de la RS por tipo de empleado. El comparativo del constructo requerimientos para la aplicación de la RS sobre dos dimensiones: apoyos al conocimiento de RS, apoyos requeridos de otros organismos respecto a los dos tipos de empleado en 275 casos (250 de nivel operativo y 25 de nivel directivo), sobre las siguientes hipótesis plateadas (ver tabla 3 ).

Ho: Los requerimientos para la aplicación de la RS son independientes del tipo de empleado.

H1: Los requerimientos para la aplicación de la RS no son independientes del tipo de empleado.

Identificando el grado de significancia de acuerdo al valor de $p>0,05$ no es significativa y $\mathrm{P}<0,05$ es significativa.

Los resultados muestran que los empleados directivos y operativos en la dimensión apoyo al conocimiento de la RS se identificaron asociación moderada en cuanto asesoría y asociación muy baja en el apoyo requerido de tecnología determinando que no son significativas y no hay influencia entre las variables; no obstante, en la variable de capacitación hay asociación significativa por lo tanto hay influencia; en la dimensión apoyos requeridos de otros organismos hay asociación muy fuerte en institutos de investigación y organismos empresariales, hay asociación baja en organismos públicos descentralizados y en agencias de cooperación internacional, hay asociación muy baja en cuanto al apoyo requerido del gobierno. Como eslabón del quehacer para la aplicación de la responsabilidad social se consideró para los requerimientos (apoyos externos) a diversas organizaciones públicas y privadas en la que se identifica un compromiso con la sustentabilidad como: organismos federales públicos descentralizados, empresariales, institutos de investigación y/o universidades, agencias de cooperación internacional, lo que reflejó una falta de vinculación, entre estos. 
PROMOCIÓN DE LA RESPONSABILIDAD SOCIAL EN EMPRESAS MANUFACTURERAS EXPORTADORAS DEL ESTADO DE HIDALGO PARA EL DESARROLLO REGIONAL

\section{Tabla 3}

Análisis de inferencias Chi cuadrada en los requerimientos para la comunicación de RS por tipo de empleado

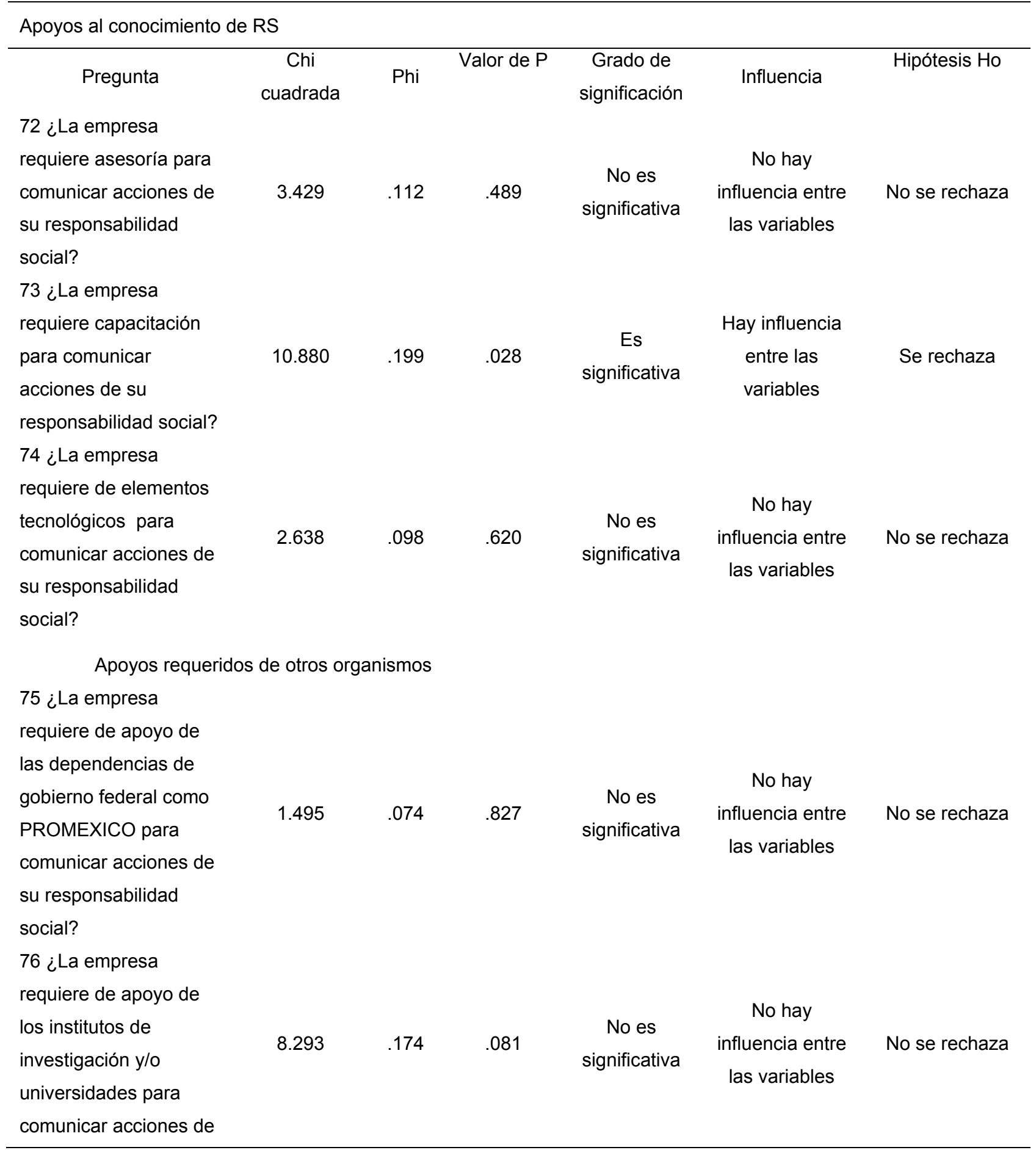


PROMOCIÓN DE LA RESPONSABILIDAD SOCIAL EN EMPRESAS MANUFACTURERAS EXPORTADORAS DEL ESTADO DE HIDALGO PARA EL DESARROLLO REGIONAL

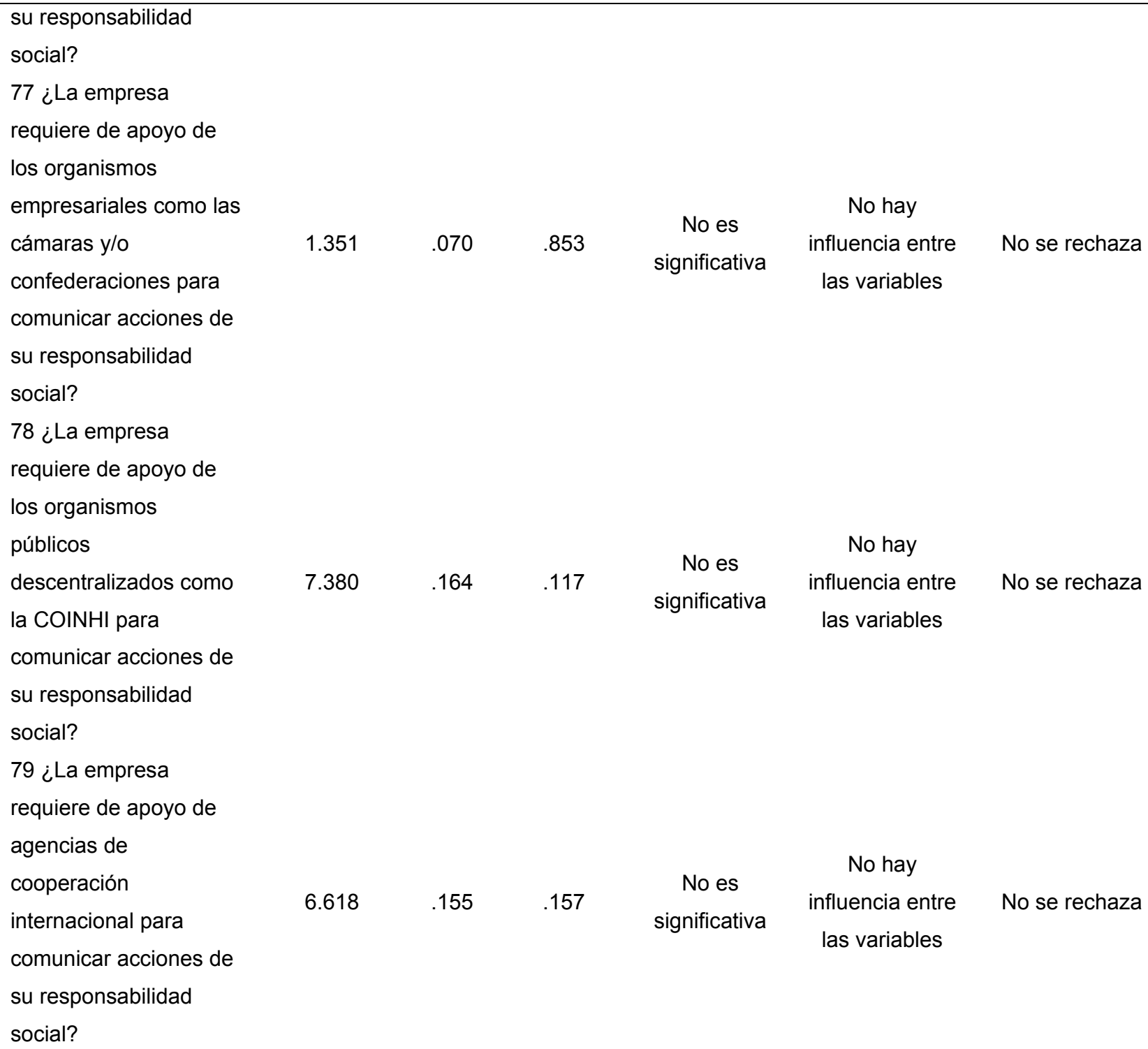

Fuente: Elaboración propia, 2017.

Propuesta: Para impulsar la responsabilidad social de las empresas manufactureras exportadoras que contribuyan al desarrollo regional del Estado de Hidalgo de manera sustentable, se propone establecer un centro de desarrollo regional sustentable, que fungirá como intermediario de vinculación con los organismos preocupados por el desarrollo sustentable, utilizando las estrategias para que apliquen la RS en las empresas, generando impacto: en el ámbito económico al generar empleos y distribuir mejor sus ingresos, en el ámbito del medio ambiente 
PROMOCIÓN DE LA RESPONSABILIDAD SOCIAL EN EMPRESAS MANUFACTURERAS EXPORTADORAS DEL ESTADO DE HIDALGO PARA EL DESARROLLO REGIONAL

el cuidado y su conservación así como, en el ámbito social al generar bienestar social, familiar, de salud, educación y cultura (ver figura 1).

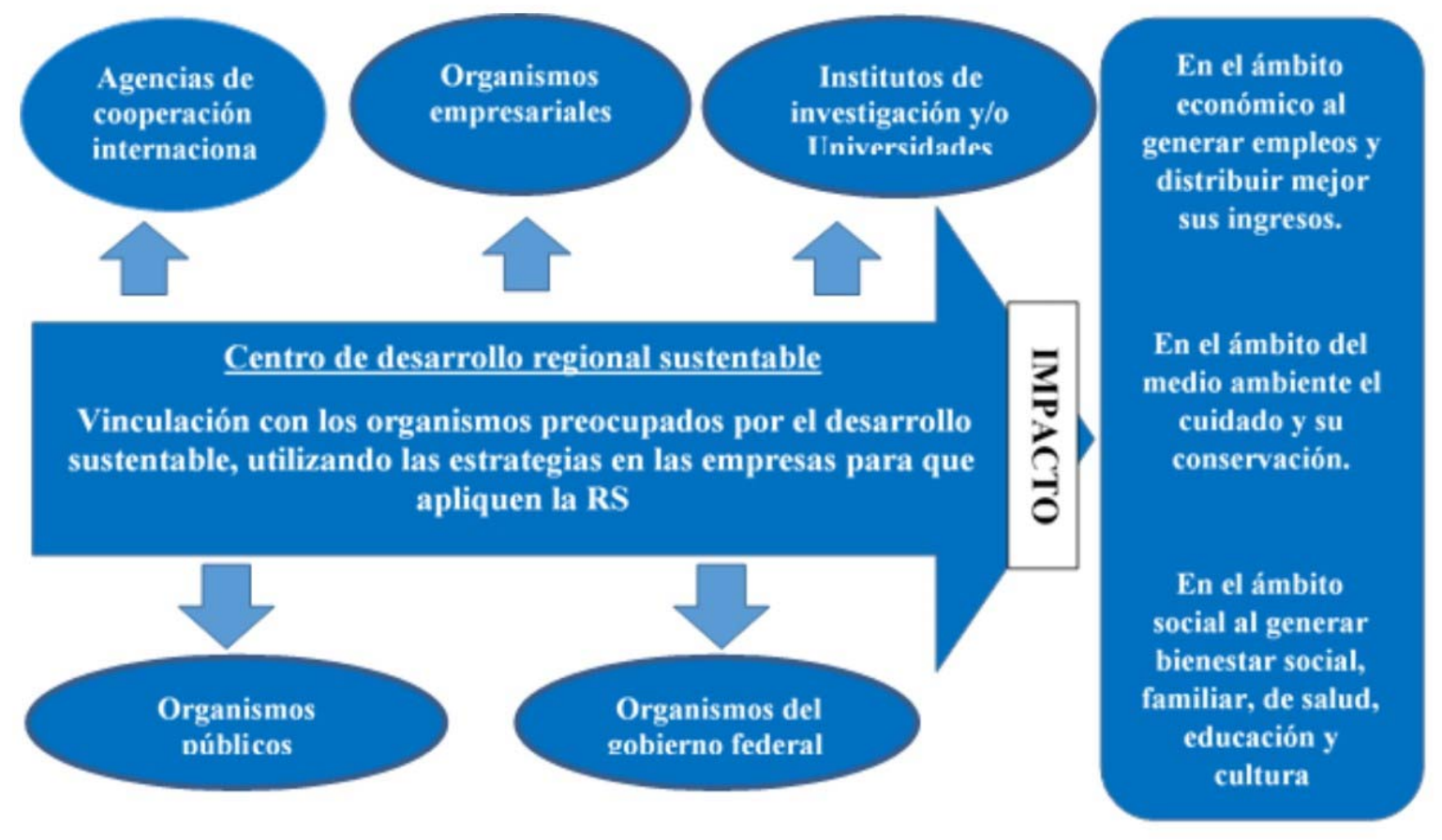

Figura 1. Promoción para implementar la responsabilidad social en las empresas manufactureras exportadoras que contribuyan al desarrollo regional del Estado de Hidalgo de manera sustentable.

Fuente: Elaboración propia, 2017.

\section{CONCLUSIONES}

Después del análisis del cuestionario aplicado a directivos y operativos de las empresas durante el segundo semestre del 2017, se puede deducir que se obtuvieron la siguientes generalidades en cuanto a determinar las necesidades de apoyo externo que requieren las empresas manufactureras exportadoras del Estado de Hidalgo para aplicar la responsabilidad social, se establece que más de la mitad consideran necesario el apoyo externo para aplicar la responsabilidad social, ubicando en el apoyo al conocimiento refiriendo asesoría, apoyos en cuanto a capacitación y elementos tecnológicos; en apoyos requeridos de otros organismos para realizar acciones de responsabilidad social, se puntualiza en cuanto a los directivos la mitad lo MUÑOZ-BAUTISTA E., CERÓN-ISLAS A., HERNÁNDEZ-ORTIZ I., LÓPEZ-GARCÍA S. E. 
PROMOCIÓN DE LA RESPONSABILIDAD SOCIAL EN EMPRESAS MANUFACTURERAS EXPORTADORAS DEL ESTADO DE HIDALGO PARA EL DESARROLLO REGIONAL

aceptan y poco menos de la mitad no lo consideran necesario, y de acuerdo a los empleados de nivel operativo, más de la mitad considera necesario el apoyo de PROMÉXICO, institutos de investigación y/o universidades, cámaras y/o confederaciones, organismos públicos descentralizados y sólo la mitad acepto el apoyo de agencias de cooperación internacional.

Dando respuesta a la hipótesis de investigación planteada al categorizar las necesidades de apoyo externo desde el punto de vista directivo y operativo que requieren las empresas manufactureras exportadoras del Estado de Hidalgo en los municipios de Pachuca de Soto, Tulancingo de Bravo, Tepeapulco y Tizayuca, en implementar la responsabilidad social de una empresa, permitió el diseño de un modelo para el impulso de la responsabilidad social en empresas manufactureras exportadoras del Estado de Hidalgo el cual se desarrolla con el soporte de los diferentes organismos interesados en la sustentabilidad los cuales se les genera una estrategia para tener un impacto favorable dentro de la sociedad, en la economía y cuidado al medio ambiente de manera sustentable.

\section{REFERENCIAS BIBLIOGRÁFICAS}

Calvo, C. (2006). Las diferentes teorías que sustentan la responsabilidad social de la empresa: Estado de situación y prospectiva. Recuperado de: http://www.cyta.com.ar/biblioteca/bddoc/bdlibros/334_calvo.pdf

RENUDIRSE, Red Iberoamericana de Universidades por la Responsabilidad Social Empresarial (RSE). Retomado de: http://www.redunirse.org/_index.php/?q=node/58

Cámara Nacional de la Industria de Transformación [CANACINTRA], (2013). Que es CANACINTRA. Recuperado de: http://www.canacintra.org.mx/index.php?option=com_content\&view=article\&id=115\&ltemid $=164$

Delgadillo, J. Torres, F. y Gazca, J. (2001). Vigencia y actualidad del desarrollo regional en México. UNAM, Casa Juan Pablos S.A. de C.V. México.

Devore, J. (2001). Probabilidad y estadística para ingeniería y ciencias (5ª ed.). México: Thomson Learning. 
PROMOCIÓN DE LA RESPONSABILIDAD SOCIAL EN EMPRESAS MANUFACTURERAS EXPORTADORAS DEL ESTADO DE HIDALGO PARA EL DESARROLLO REGIONAL

Diario Oficial de la Federación [DOF] (2012). Declaratoria de vigencia de la Norma Oficial Mexicana NMX-SAST-26000-IMNC-2011. Retomado de: http://legismex.mty.itesm.mx/normas/IMNC/DecVigNMX-sast-26000-IMNC-2011-0612.pdf y en http://diariooficial.segob.gob.mx/

Díaz, N. (2011). Certificación ineludible. Revista Gestión. 11(3).

Flores, S. (2012). Como se define el desarrollo regional. Apuntes de la materia entorno social y nuevos esquemas de desarrollo. UPAEP primavera 2012.

Global Reporting Initiative GRI (2006). Guía para la elaboración de Memorias de Sostenibilidad. Recuperado de: https://www.globalreporting.org/resourcelibrary/Spanish-G3-Complete.pdf

Hernández, R., Fernández, C., \& Baptista, M. (2010). Metodología de la Investigación (5ª ed.). Chile: McGraw Hill.

Instituto Mexicano de Normalización y Certificación [IMNC], (2012). Responsabilidad Social Empresarial, Mapeo de Promotores. Recuperado de: http://www.mapeorse.info/promotor/instituto-mexicano-de-normalizaci\%C3\%B3n-y-certificaci\%C3\%B3n-imnc

International Organization for Standardization [ISO]. (2009). Environmental management The ISO 14000 family of International Standards. Recuperado de: http://www.iso.org/iso/theiso14000family_2009.pdf, http://www.iso.org/iso/home.html.

Keegan, W., \& Green, M. (2009). Fundamentos de mercadotecnia internacional. México: Pearson Educación.

Korin M. (2013). Agencia de Cooperación Técnica Alemana (GTZ). Mapeo de promotores de RSE América Latina. Recuperado de: http://www.mapeo-rse.info/promotor/agencia-decooperaci\%C3\%B3n-t\%C3\%A9cnica-alemana-gtz

KPMG International Cooperative, (2011). International Survey of Corporate Responsibility Reporting 2011. Autor. Recuperado de: http://comunicarseweb.com.ar/download.php?tipo=acrobat\&view=1\&dato=1320672202_CR _Report_2011.pdf 
PROMOCIÓN DE LA RESPONSABILIDAD SOCIAL EN EMPRESAS MANUFACTURERAS EXPORTADORAS DEL ESTADO DE HIDALGO PARA EL DESARROLLO REGIONAL

Lugo, L. (2011). Programa Institucional de la Corporación Internacional Hidalgo COINHI), 20112016. Hidalgo. Gob. Recuperado de: http://intranet.ehidalgo.gob.mx/siieh/Programas\%20Sectoriales/files/programa_institucional_coinhi.pdf

Lindgreen, A., Swaen, V. \& Johnston, W. (2009). The supporting function of marketing in corporate social responsibility. Corporate Reputation Review. 12(2), pp. 120-139.

Malhotra, N. (2008). Investigación de mercados. México: Pearson Prentice Hall.

Muñoz \& López, (2013). Las empresas exportadoras del Estado de Hidalgo y el desarrollo sustentable. Una revisión al ser ESR. México: UAEH.

Pedroza, A. \& Ahuja L. (2008). La responsabilidad social empresarial: del modelo Alemán al contexto mexicano. Una recuperación del debate. México: Fundación Konrad Adenauer.

Porter, M. (2008). On Competition. E.U. A.: Harvard Business Review Book.

PROMÉXICO (2013). Desarrollo sustentable y el crecimiento económico en México. Recuperado de: http://www.promexico.gob.mx/desarrollo-sustentable/

Rachman, D. (1996). Introducción a los negocios. México: McGraw Hill.

Salguero, J. (2006). Enfoques sobre algunas teorías referentes al desarrollo regional. Sociedad geográfica de Colombia. Academia de Ciencias Geográficas. Recuperado de: http://www.economia.unam.mx/academia/inae/inae5/515.pdf

Trejo, L. (2012). Elabora Coparmex ley para responsabilidad social empresarial. El Independiente. Recuperado de: www.elindependientedehidalgo.com.mx/archivos/51204

UAEH (2013). Servicios. Dirección de vinculación con el sector social y productivo. Recuperado de: http://www.uaeh.edu.mx/adminyserv/gesuniv/div_vin/dir_socprod/servicios.html

UAE, (2014). Misión, visión. Dirección de servicio social y prácticas profesionales. Recuperado de: http://www.uaeh.edu.mx/adminyserv/gesuniv/div_vin/dir_sspract/mision.html

World Confederation of Businesses [WORLDCOB] (2011). Norma WORLDCOB-CSR: 2011.1. Recuperado de: http://www.worldcob-csr.com/es/norma.php 